\title{
On dynamic interaction between mechanical systems and selected electric motors
}

\author{
Tomasz Szolc ${ }^{1, *}$, Robert Konowrocki ${ }^{1}$, and Andrzej Pochanke ${ }^{2}$ \\ ${ }^{1}$ Institute of Fundamental Technological Research of the Polish Academy of Sciences, \\ ul. Pawińskiego 5B, 02-106 Warsaw, Poland \\ ${ }^{2}$ Warsaw University of Technology, Faculty of Electric Engineering, \\ Plac Politechniki 1, 00-661 Warsaw, Poland
}

\begin{abstract}
In the paper there is presented a reliable structural model of the rotating mechanical systems as well as mathematical models of the stepping, synchronous and asynchronous motors, by means of which electromechanical coupling effects can be thoroughly investigated. An importance and severity of these phenomena, not sufficiently explored till present, have been demonstrated by results obtained for transient and steady-state operational conditions in the computational examples concerning torsional vibrations of drive trains with various electric motors.
\end{abstract}

\section{Introduction}

During last few decades electric motors became more and more commonly applied for driving of various machines, mechanisms and vehicles. Usually, such sources of power are characterized by simpler structures, they are more robust, efficient, precise to control and environmental friendly than e.g. piston and flow internal combustion engines or hydraulic motors. Nowadays, modern asynchronous, synchronous and stepping motors are particularly popular to drive mechanical objects with various geometrical dimensions, weights, rotational speeds and levels of power consumption. Because of this reason, electromechanical interactions with just these kinds of motors have been studied till present by many authors from numerous research centres in the whole world, e.g. in [1-5]. But still in majority of works mechanical engineers focus their attention on detailed dynamic properties of mechanical systems and they take into consideration an interaction of an electric motor in the form of 'a priori' assumed electromagnetic driving torque functions. Electrical engineers, however, very thoroughly model electric motors and treat the driven mechanical objects in very simplified forms of few degrees-of-freedom dynamic oscillators or even single rigid bodies. But by the use of such approaches many important electromechanical interaction effects occurring in steady-state and transient operational conditions cannot be considered. Namely, then it is very difficult to investigate an influence of motor rotor rotational speed fluctuations on oscillations of electric currents in the motor windings and, consequently, on fluctuations of the electromagnetic driving torque which is responsible for excitation of torsional vibrations of a mechanical system.

* Corresponding author: tszolc@ippt.pan.pl 
In this paper dynamic interactions between mechanical systems in the form of rotating machines with working tools driven by electric motors by means of sets of stepped shafts, couplings and gear stages will be studied. As drive sources a steeping motor, permanent magnet synchronous motor (PMSM) and asynchronous motor are going to be considered.

\section{Hybrid structural modelling of mechanical systems}

In order to investigate a detailed character of the electromechanical coupling, the possibly realistic and reliable mechanical model of the driven system is applied. In this paper, similarly as e.g. in [6,7], dynamic investigations of the entire driven system are performed by means of the one-dimensional hybrid structural model consisting of finite continuous visco-elastic macro-elements and rigid bodies. In this model by the torsionally deformable cylindrical macro-elements of continuously distributed inertial-visco-elastic properties there are substituted successive cylindrical segments of the stepped shafts and coupling disks. In order to obtain a sufficiently accurate representation of the real object, the visco-elastic macro-elements in the hybrid model are characterized by the geometric cross-sectional polar moments of inertia responsible for their elastic and inertial properties as well as by the separate layers responsible for their inertial properties only. The inertias of gear-wheels and driven machine working tools are represented by rigid bodies attached to the appropriate macro-element cross-sections.

In the hybrid model torsional motion of cross-sections of each visco-elastic macroelement is governed by the local hyperbolic partial differential equations of the wave type. Mutual connections of the successive macro-elements creating the stepped shaft as well as their interactions with the rigid bodies are described by equations of boundary conditions. These equations contain geometrical conditions of conformity for rotational displacements of the extreme cross sections of the mutually adjacent elastic macro-elements as well as linear equations of equilibrium for external torques as well as for inertial, elastic and external damping moments.

The solution for forced vibration analysis has been obtained using the analytical computational approach described e.g. in [6,7]. Solving the differential eigenvalue problem and an application of the Fourier solution in the form of series in the orthogonal eigenmode functions lead to the set of modal equations for time coordinates $\xi_{m}(t)$ :

$$
\ddot{\xi}_{m}(t)+\left(\beta+\tau \omega_{m}^{2}\right) \dot{\xi}_{m}(t)+\omega_{m}^{2} \xi_{m}(t)=\frac{1}{\gamma_{m}^{2}}\left(X_{m}^{S} \cdot T_{e l}(t)-X_{m}^{R} \cdot M_{r}(t)\right), \quad m=1,2, \ldots
$$

where $\omega_{m}$ are the successive natural frequencies of the drive system, $\beta$ denotes the coefficient of external damping assumed here as proportional one to the modal masses $\gamma_{m}^{2}$, $\tau$ is the retardation time, $T_{e l}(t)$ denotes the external torque generated by the electric motor, $M_{r}(t)$ is the driven machine retarding torque and $X_{m}^{S}, X_{m}^{R}$ are the modal displacements scaled by proper maxima and corresponding respectively to the electric motor- and to the driven machine working tool-locations in the hybrid model. A fast convergence of the applied Fourier solution enables us to reduce the number of the modal equations to solve in order to obtain a sufficient accuracy of results in the given range of frequency. Here, it is necessary to solve only a few or at most a dozen or so modal equations (1), even in cases of very complex mechanical systems, contrary to the classical one-dimensional beam finite element formulation leading usually to large numbers of motion equations corresponding each to more than one hundred or many hundreds degrees of freedom (if the artificial and often error-prone model reduction algorithms are not applied). 


\section{Modelling of the electric motors}

From the viewpoint of electromechanical coupling investigation properly advanced circuit models of the electric motors seem to be sufficiently accurate.

\subsection{Modelling of the stepping motor}

According e.g. to [5], the mathematical model of a typical four-cycle, double-phase stepping motor with the fundamental step angle $1.8 \mathrm{deg}=0.0314 \mathrm{rad}$, which means that its rotor is characterized by $Z_{r}=50$ salient poles, is described by two voltage equations:

$$
\begin{gathered}
L \frac{\mathrm{d} i_{1}(t)}{\mathrm{d} t}+R i_{1}(t)-K_{\mathrm{U}} \frac{\mathrm{d} \Theta(t)}{\mathrm{d} t} \cdot \sin (\tilde{\Theta}(t))=-U(t) \cdot \operatorname{sgn}\{\sin (\Phi(t))\}, \\
L \frac{\mathrm{d} i_{2}(t)}{\mathrm{d} t}+R i_{2}(t)+K_{\mathrm{U}} \frac{\mathrm{d} \Theta(t)}{\mathrm{d} t} \cdot \cos (\tilde{\Theta}(t))=U(t) \cdot \operatorname{sgn}\{\cos (\Phi(t))\}, \Phi(t)=\frac{\pi}{2} \int_{0}^{t} f(\tau) \mathrm{d} \tau,
\end{gathered}
$$

where $i_{1}(t), i_{2}(t)$ denote the electric currents in both motor phases, $L$ is the phase inductance, $R$ denotes the resistance of one phase, $K_{\mathrm{U}}$ is the motor voltage constant, $\Theta(t)$ denotes the instantaneous rotation angle of the rotor including the rigid body motion and the vibratory component, $U(t)$ is the slowly varying, controlled supply voltage, $\tilde{\Theta}(t)$ denotes the rotor electric angle, which in the case of the stepping motor can be determined as $\tilde{\Theta}(t)=Z_{r} \Theta(t)$, and $f(t)$ is the voltage supply commutation frequency. Here, a sufficiently good commutation realized by means of a proper stepping motor control should result in the control voltage supply phase angle $\Phi(t) \cong \tilde{\Theta}(t)$. The electromagnetic torque generated by the double-phase stepping motor is expressed by the following formula:

$$
T_{e l}(t)=K_{\mathrm{T}}\left[-i_{1}(t) \cdot \sin (\tilde{\Theta}(t))+i_{2}(t) \cdot \cos (\tilde{\Theta}(t))\right],
$$

where $K_{\mathrm{T}}$ denotes the stepping motor torque constant. By substituting expression (3) into the modal equations (1) and upon a proper combinations of them with the voltage equations (2) one obtains the coupled set the parametric ordinary differential equations:

$$
\mathbf{M} \ddot{\mathbf{r}}(t)+\mathbf{C}(\tilde{\Theta}(t)) \cdot \dot{\mathbf{r}}(t)+\mathbf{K}(\tilde{\Theta}(t)) \cdot \mathbf{r}(t)=\mathbf{F}(t, \dot{\mathbf{r}}(t)),
$$

where: $\mathbf{C}(\tilde{\Theta}(t))=\mathbf{C}_{0}+\mathbf{C}_{\mathrm{E}}(\tilde{\Theta}(t)), \quad \mathbf{K}(\tilde{\Theta}(t))=\mathbf{K}_{0}+\mathbf{K}_{\mathrm{E}}(\tilde{\Theta}(t))$,

$$
\mathbf{r}(t)=\operatorname{col}\left[i_{1}(t), i_{2}(t), \xi_{0}(t), \xi_{1}(t), \xi_{2}(t), \ldots\right] .
$$

The symbols $\mathbf{M}, \mathbf{C}_{0}$ and $\mathbf{K}_{0}$ denote, respectively, the constant diagonal modal mass, damping and stiffness matrices, $\mathbf{C}_{\mathrm{E}}(\tilde{\Theta}(t))$ is the band matrix of the inductive-electromagnetic effects and $\mathbf{K}_{\mathrm{E}}(\tilde{\Theta}(t))$ denotes the band matrix of the resistant-electro-magnetic effects, both of harmonically variable coefficients with the frequency following from the current electric rotation angle. The symbol $\mathbf{F}(t, \dot{\mathbf{r}}(t))$ denotes the external excitation vector due to the control input voltage and the retarding torques. The unknown co-ordinate vector $\mathbf{r}(t)$ consists of the electric currents in both motor phases and of the unknown time functions $\xi_{m}(t)$ in the Fourier solutions, $m=0,1,2, \ldots$. In order to obtain the system's dynamic response, equations (4) are solved by means of a direct integration. The number of equations (4) corresponds to the number of eigenmodes taken into consideration in the range of frequency of interest. These equations are mutually coupled by the parametric terms expressing the electromagnetic interaction with the stepping motor and they are very convenient for effective numerical simulations of a dynamic behaviour of the electromechanical system. 


\subsection{Modelling of the permanent magnet synchronous motor (PMSM)}

In the case of a permanent magnet symmetrical, 3-phase synchronous motor with a uniform air-gap distribution and stator windings star-connected, voltage equations formulated in the natural system of electrical exes "a-b-c" have the following form:

$$
\begin{aligned}
& L \frac{\mathrm{d} i_{a}(t)}{\mathrm{d} t}+M \frac{\mathrm{d} i_{b}(t)}{\mathrm{d} t} M \frac{\mathrm{d} i_{c}(t)}{\mathrm{d} t}+R i_{a}(t)-\psi \frac{\mathrm{d} \tilde{\Theta}(t)}{\mathrm{d} t} \cdot \sin (\tilde{\Theta}(t))=U(t) \sin (\tilde{\Theta}(t)) \\
& L \frac{\mathrm{d} i_{b}(t)}{\mathrm{d} t}+M \frac{\mathrm{d} i_{a}(t)}{\mathrm{d} t}+M \frac{\mathrm{d} i_{c}(t)}{\mathrm{d} t}+R i_{b}(t)-\psi \frac{\mathrm{d} \tilde{\Theta}(t)}{\mathrm{d} t} \sin \left(\tilde{\Theta}(t)-\frac{2}{3} \pi\right)=U(t) \sin \left(\tilde{\Theta}(t)-\frac{2}{3} \pi\right) \\
& L \frac{\mathrm{d} i_{c}(t)}{\mathrm{d} t}+M \frac{\mathrm{d} i_{a}(t)}{\mathrm{d} t}+M \frac{\mathrm{d} i_{b}(t)}{\mathrm{d} t}+R i_{c}(t)-\psi \frac{\mathrm{d} \tilde{\Theta}(t)}{\mathrm{d} t} \sin \left(\tilde{\Theta}(t)+\frac{2}{3} \pi\right)=U(t) \sin \left(\tilde{\Theta}(t)+\frac{2}{3} \pi\right)
\end{aligned}
$$

where $\left.i_{\mathrm{a}}(t), i_{\mathrm{b}}(t)\right), i_{\mathrm{c}}(t)$ denote the electric currents in motor phases, $M$ denotes the mutual stator coil inductance and $\psi$ is the flux generated by the permanent magnet. The remaining symbols have been already defined above. Upon the similar combination as that performed in the case of the stepping motor one obtains the analogous to (4) coupled set the parametric ordinary differential equations. In order to carry out qualitative analyses, using Clark-Park's transformation for the currents and voltages in Eqs. (5), one obtains:

$$
\left[\begin{array}{ccc}
R+(L-M) \frac{\mathrm{d}}{\mathrm{d} t} & (L-M) \frac{\mathrm{d} \tilde{\Theta}(t)}{\mathrm{d} t} & 0 \\
-(L-M) \frac{\mathrm{d} \tilde{\Theta}(t)}{\mathrm{d} t} & R+(L-M) \frac{\mathrm{d}}{\mathrm{d} t} & -\psi \frac{\mathrm{d} \tilde{\Theta}(t)}{\mathrm{d} t}
\end{array}\right] \cdot\left[\begin{array}{c}
i_{d} \\
i_{q} \\
1
\end{array}\right]=\left[\begin{array}{c}
u_{d} \\
u_{q}
\end{array}\right] .
$$

The above equations make the coupling between the electrical and mechanical part nonlinear. Upon Clark-Park's transformation the same property has a circuit model of the asynchronous motor. Thus, according to [4], qualitative analyses carried out for both kinds of electric motors can lead to a determination of rotor-to-stator electromagnetic rotational stiffness- and damping characteristics using the harmonic balance method to solve analytically the voltage equations transformed into mutually perpendicular electric axes.

\subsection{Modelling of the asynchronous motor}

In the case of the symmetrical three-phase asynchronous motor electric current oscillations in its windings are described by the six circuit voltage equations transformed next into the system of four Park's equations in the so called ' $\alpha \beta-d q$ ' reference system, [4]:

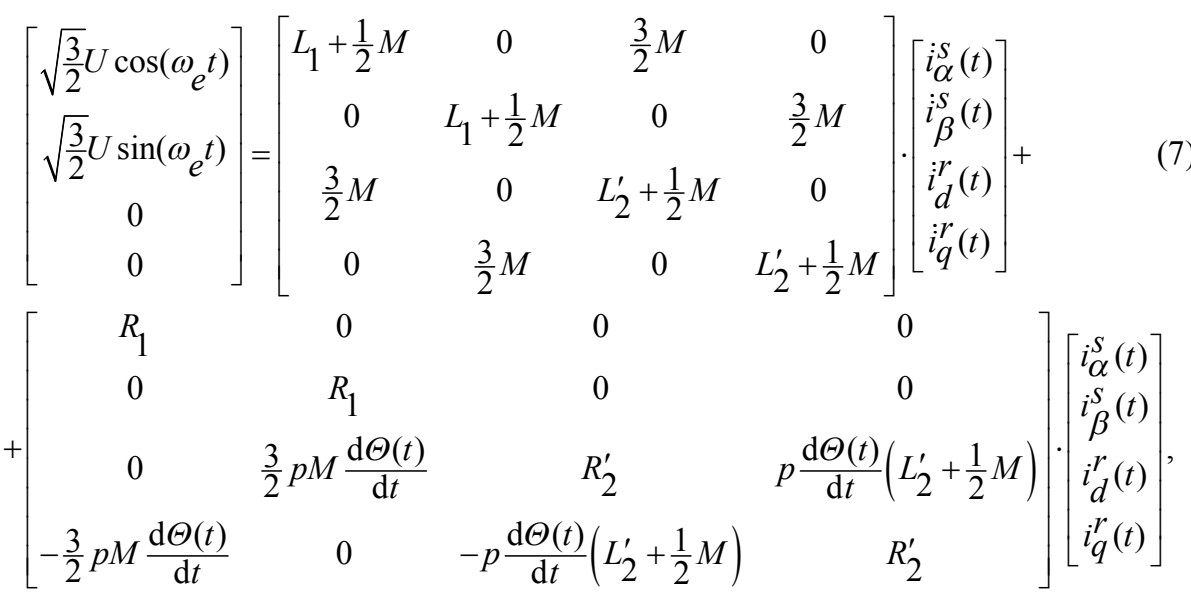


where $\omega_{e}$ is the supply voltage circular frequency, $L_{1}, L_{2}$ ' are the stator coil inductance and the equivalent rotor coil inductance, respectively, $M$ denotes the relative rotor-to-stator coil inductance, $R_{1}, R_{2}$ ' are the stator coil resistance and the equivalent rotor coil resistance, respectively, $p$ is the number of pairs of the motor magnetic poles and $i_{\alpha}{ }^{s}, i_{\beta}{ }^{s}$ are the electric currents in the stator windings reduced to the electric field equivalent axes $\alpha$ and $\beta$ and $i_{d}^{r}$, $i_{q}{ }^{r}$ are the electric currents in the rotor windings reduced to the electric field equivalent axes $d$ and $q$, [4]. Then, the total electromagnetic torque and its oscillatory part generated by such a motor can be expressed by the following formulae:

$$
T_{e l}=\frac{3}{2} p M\left(i_{\beta}^{S} \cdot i_{d}^{r}-i_{\alpha}^{s} \cdot i_{q}^{r}\right), \quad T_{e l}^{\mathrm{var}}(t)=S(\omega) \cdot \sin (\omega t)+T(\omega) \cdot \cos (\omega t) .
$$

By projecting the sine- $S(\omega)$ and cosine- $T(\omega)$ components of the electromagnetic torque oscillatory part as well as the analogous components of the rotor rotation angle, respectively, on the complex plane real and imaginary axes and using the proper definitions given e.g. in [3], the electromagnetic torsional stiffness $k_{e}(\omega)$ and the coefficient of damping $d_{e}(\omega)$ generated by the asynchronous motor are determined in the following form:

$$
k_{e}(\omega)=-\frac{U \cdot S(\omega)+W \cdot T(\omega)}{U^{2}+W^{2}}, \quad d_{e}(\omega)=-\frac{1}{\omega} \cdot \frac{U \cdot T(\omega)-W \cdot S(\omega)}{U^{2}+W^{2}},
$$

$$
\text { where: } \begin{aligned}
W & =\sum_{m=0}^{\infty} \frac{\left(X_{m}^{S}\right)^{2} T(\omega) \cdot\left(\omega_{m}^{2}-\omega^{2}\right)-\left[\left(X_{m}^{S}\right)^{2} S(\omega)-X_{m}^{S} X_{m}^{R} R\right] \cdot\left(\beta+\tau \omega_{m}^{2}\right) \omega}{\gamma_{m}^{2}\left[\left(\omega_{m}^{2}-\omega^{2}\right)^{2}+\left(\beta+\tau \omega_{m}^{2}\right)^{2} \omega^{2}\right]}, \\
U & =\sum_{m=0}^{\infty} \frac{\left[\left(X_{m}^{S}\right)^{2} S(\omega)-X_{m}^{S} X_{m}^{R} R\right] \cdot\left(\omega_{m}^{2}-\omega^{2}\right)+\left(X_{m}^{S}\right)^{2} T(\omega) \cdot\left(\beta+\tau \omega_{m}^{2}\right) \omega}{\gamma_{m}^{2}\left[\left(\omega_{m}^{2}-\omega^{2}\right)^{2}+\left(\beta+\tau \omega_{m}^{2}\right)^{2} \omega^{2}\right]} .
\end{aligned}
$$

The above expressions derived in the framework of a qualitative analysis of properties of the asynchronous motor, that drives a linear model of the rotating machine, enable us to carry out thorough dynamic investigations of the coupled electromechanical system.

\section{Computational examples}

In the first computational example there is performed a simulation of the run-up, steady state operation and run-down of the precise geared drive system driven by the stepping motor. A mechanical model of this object is shown in Fig. 1. It has been uniformly accelerated from its standstill to the constant average rotational speed $n=210 \mathrm{rpm}$ and nominal torque $0.35 \mathrm{Nm}$ within $3 \mathrm{~s}$ in order to operate for next $1 \mathrm{~s}$ under the constant retarding torque generated by the power receiver. Then, within successive $3 \mathrm{~s}$ the drive system was uniformly stopped back to the standstill. In Fig. 2 in time and frequency domain there are presented plots of the considered system dynamic response obtained for two simulation modes: for the "coupled mode", where the results have been determined by means of an integration of Eqs. (4) and marked by the black lines, as well as for the traditionally applied "uncoupled mode" illustrated here by the grey lines. In the latter case the mechanical system was treated as a one rigid body, i.e. only modal co-ordinate $\xi_{0}$ in (4) has been taken into consideration in order to determine the motor electromagnetic torque (5) to be imposed next as an external excitation of the hybrid mechanical model of the drive system. It is worth noting that the results obtained for these two modes are significantly different from each other both in transient and steady-state operational conditions. During the start-up and run-down the rotational speeds as well as the dynamic torques observed in 


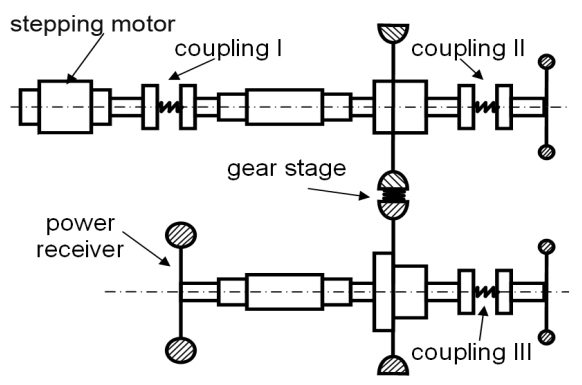

Fig. 1. The hybrid mechanical model of the precise drive system.

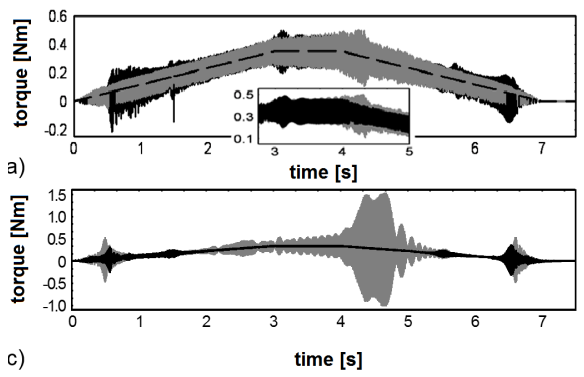

the system input and output shaft are characterized by severe transient resonances with the fundamental eigenmode of the first natural frequency equal $103.4 \mathrm{~Hz}$, as shown in Figs. 2b-d. The amplitudes of this resonances are particularly severe in the case of the uncoupled mode, although in these operational conditions the coupled mode yields much more severe external excitation produced by the driving motor, which follows from Fig. 2a. However, during the steadystate operation in the case of uncoupled mode
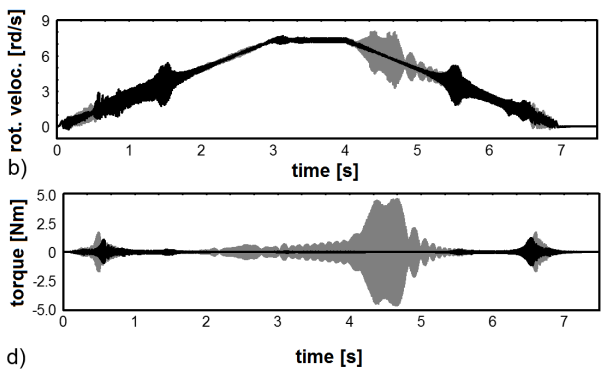

d)

time [s]

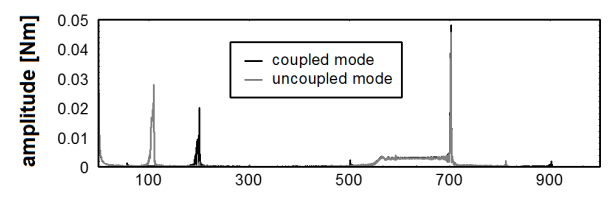

e)

frequency $[\mathrm{Hz}]$

Fig. 2. Dynamic response of the electromechanical system: time histories of the electromagnetic motor torque (a), rotational speed of the output shaft (b), dynamic torques transmitted by the input (c) and output (d) shaft, and the amplitude spectra of the stepping motor torque (e).

the abovementioned regular and slightly stronger electromagnetic excitation results in resonant responses both for the rotational speeds and the dynamic torques. In order to explain this fact the FFT analysis of the time-histories of the excitation torque generated by the stepping motor has been performed for the coupled and uncoupled mode. The amplitude spectra obtained for the coupled and uncoupled mode and depicted in Fig. 2e are characterized by almost identical the greatest peaks of frequency $700 \mathrm{~Hz}$ corresponding to the fundamental excitation component produced by the stepping motor in the steady-state operation conditions. This value results from the assumed nominal rotational speed $n=210$ rpm and the fundamental step angle $0.0314 \mathrm{rad}$ of the considered motor. In addition to these peaks with frequency of $700 \mathrm{~Hz}$, different sub-harmonic components of the excitation torque are generated. In the case of uncoupled mode, in Fig. 2e the significant excitation peak of frequency ca. $100 \mathrm{~Hz}$ is observed, which is very close to the first drive system natural frequency equal to $103.4 \mathrm{~Hz}$. This component is responsible for the observed resonance effects depicted in Figs. $2 b-d$ for the uncoupled mode, in a contradistinction to the coupled mode, where the analogous sub-harmonic excitation component is characterized by frequency $\sim 200 \mathrm{~Hz}$ which is far away from the first and the second system natural frequency equal to $344.4 \mathrm{~Hz}$. According to the above, one can conclude that the taken into consideration vibratory, inertial-visco-elastic properties of the mechanical system essentially influence qualitatively and quantitatively the electromechanical coupling effects resulting respectively in different dynamic responses. 
In the second computational example the dynamic investigations will be carried out for a rotor machine with a typical structure for blowers, compressors, pumps or high-speed beater mills. Namely, their rotor-shaft contains a heavy impeller attached to the drive shaft in an overhung form and is driven by the $200 \mathrm{~kW}, 400 \mathrm{~V}$ Y asynchronous motor with the nominal rotational speed of $2980 \mathrm{rpm}$ through a flexible coupling with a non-linear characteristic. There are also worth noting the characteristic features of such device, i.e. a huge polar mass moment of inertia of the whole driven part in comparison with the polar mass moment of inertia of the motor rotor as well as a much smaller torsional stiffness of the elastic coupling than torsional stiffness values of all rotor-shaft segments. The hybrid model of this object is presented in Fig. 3.

In Fig. 4a there are shown static characteristics of the considered asynchronous motor determined according to [4] for four variants of the starting torque values which depend on the rotor resistance: namely, the greater the resistance, the greater the starting torque. In Fig. $4 b$ characteristics of the electromagnetic stiffness and coefficients of damping generated by the considered asynchronous

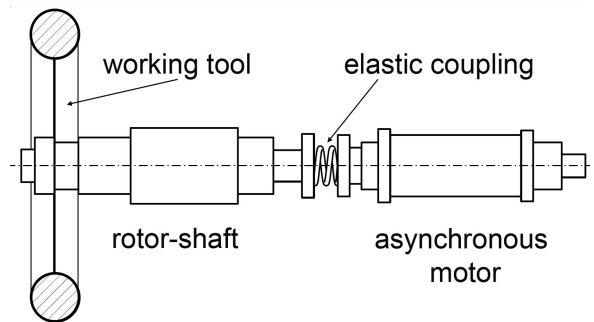

Fig. 3. The hybrid mechanical model of the rotor machine drive system.
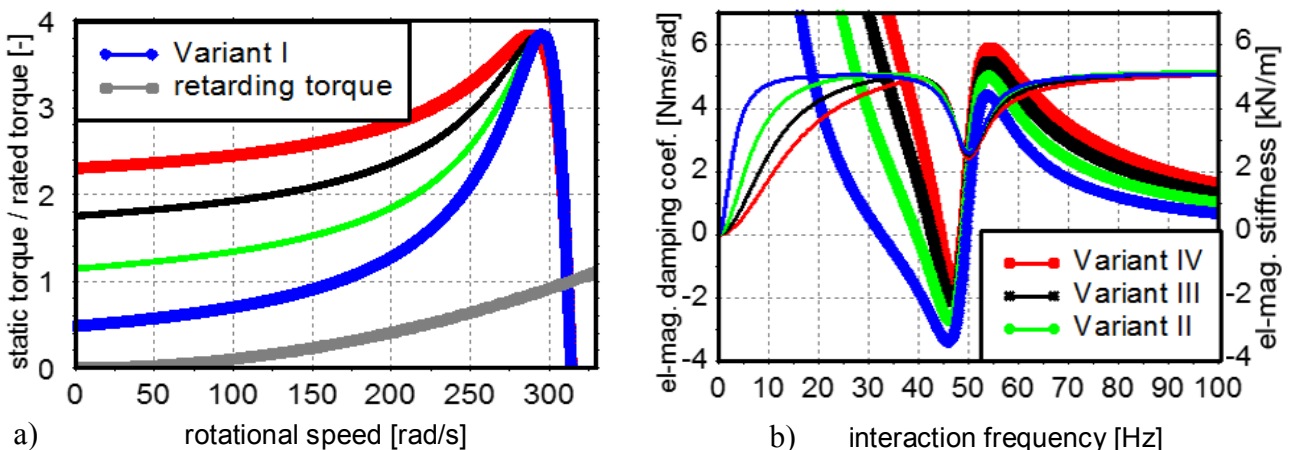

b) interaction frequency $[\mathrm{Hz}]$

Fig. 4. Static characteristics of the asynchronous motor and of the retarding torque (a) and dynamic characteristics of the rotor-to-stator electromagnetic damping and stiffness (b).

motor within the torsional interaction frequency range of $0-100 \mathrm{~Hz}$ are plotted. In this figure the thin lines correspond to the stiffness characteristics and the thick lines, appropriately, to the damping ones. These characteristics have been determined by means of relationships (9). Using respectively the same colours, these plots correspond to the mentioned above four variants of rotor resistances and thus to four variants of motor static characteristics presented in Fig. 4a. It is to emphasize that all the electromagnetic damping characteristics indicate negative value zones. From Figs. $4 \mathrm{a}$ and $4 \mathrm{~b}$ it follows that for the considered here four starting motor torque variants the breadths of these negative damping zones gradually decrease with the rise of the starting motor torque values.

In order to demonstrate a danger created by the negative electromagnetic damping generated by the asynchronous motor, start-ups of the considered rotor machine to the rotational speed of $2400 \mathrm{rpm}$ within $60 \mathrm{~s}$ have been performed. Then, for the aim of examination of a sensitivity of the considered drive train to operational instabilities, after next $12 \mathrm{~s}$ of the rotor machine steady state motion a slight step-wise increase of the retarding torque was assumed. For such an operation scenario the run-up simulation results are depicted in Fig. 5a-b. In the face of commonly observed very weak structural damping 

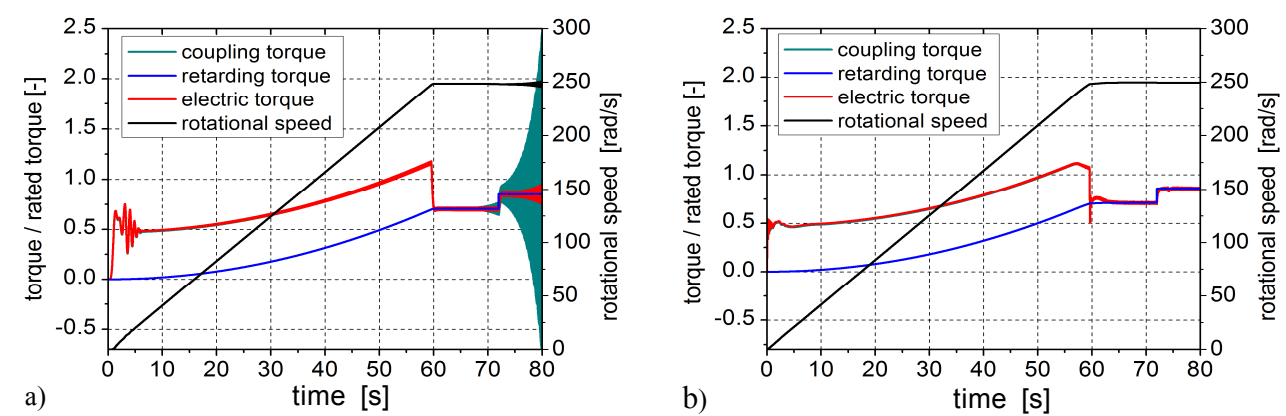

Fig. 5. Torsional response due to the start-up using the open-loop scalar control (a) and by means of the closed-loop PID vector control (b) of the starting motor torque.

in torsionally vibrating systems an application of the open-loop scalar control ' $U / f=$ const' of the asynchronous motor results in instability caused by the negative electromagnetic damping, as shown in Fig. 5a. Here, the rotational speed $2400 \mathrm{rpm}$ corresponds to a synchronous excitation of $40 \mathrm{~Hz}$ which is contained in the negative damping zone of the motor starting torque Variant I depicted in Fig. 4b. Then, due to the existing deficiency of resultant positive damping in the system, free torsional vibrations with its first natural frequency of $34.7 \mathrm{~Hz}$ and rapidly increasing amplitudes are induced. If in the considered system the negative electromagnetic damping cannot be naturally compensated by the mechanical one, a proper closed-loop control of the asynchronous motor is able to prevent such instability. In Fig. $5 \mathrm{~b}$ there are presented time-histories of a dynamic response obtained for exactly the same system parameters as before, but for the PID control realized by means of vector inverter with gains determined using Ziegler-Nichols' or Cohen-Coon's methods. The both approaches result in the visible stabilization of the considered object.

\section{Final remarks}

The performed study confirms that dynamic interaction between electric motors and mechanical systems can be thoroughly investigated when sufficiently reliable physical and mathematical models of these objects are applied. From the computational results it follows that torsional vibrations of the driven mechanical system essentially influence qualitatively and quantitatively its excitation by the stepping motor. Furthermore, negative damping generated by the asynchronous motor is responsible for operational instability of the entire drive train and by means of a proper motor control this dangerous effect can be eliminated.

\section{References}

1. B.F. Evans, A.J. Smalley, H.R. Simmons, ASME Paper, 85-DET-122, (1985)

2. A. Tabesh, R. Iravani, IEEE Trans. Energy Convers. 20 (2), 268-275, (2005)

3. T.P. Holopainen, A.-K. Repo, J. Järvinen, Proc. of the 8th IFToMM Int. Conf. on Rotordynamics (KIST, Seoul, Korea, 2010), 986-993.

4. T. Szolc, R. Konowrocki, M. Michajłow, A. Pręgowska, MSSP, 49, 118-134, (2014)

5. R. Konowrocki, T. Szolc, A. Pochanke, A. Pręgowska, MSSP, 70-71, 397-413, (2016)

6. T. Szolc, Int. J. Rot. Mach. 6 (2), 135-149, (2000)

7. T. Szolc, A dynamic analysis of complex discrete-continuous mechanical systems. Habilitational dissertation (IFTR Reports, Warsaw 2/2003) (in Polish) 